\title{
Enhanced power conversion efficiency via hybrid ligand exchange treatment of p-type PbS quantum dots
}

\author{
Zhi Li Teh ${ }^{a}$, Long Hü ${ }^{a}$, Zhilong Zhang ${ }^{b}$, Angus R. Gentlec, Zihan Chen ${ }^{a}$, Yijun Gao ${ }^{a}$, Lin Yuan ${ }^{d}$, \\ Yicong $\mathrm{Hu}^{a}$, Tom $\mathrm{Wu}^{e}$, Robert J. Patterson ${ }^{a}$, Shujuan Huang ${ }^{a, f *}$ \\ ${ }^{a}$ Australian Centre for Advanced Photovoltaics, School of Photovoltaic and Renewable Energy Engineering, University of New South Wales \\ (UNSW), Sydney, 2052, NSW, Australia \\ ${ }^{b}$ Cavendish Laboratory, University of Cambridge, J. J. Thomson Avenue, Cambridge, CB3 OHE, United Kingdom \\ ' School of Mathematical and Physical Sciences, University of Technology Sydney, 15 Broadway, Ultimo, 2007, NSW, Australia \\ ${ }^{a}$ Department of Chemistry-Ångström, Physical Chemistry, Uppsala University, 75120 Uppsala, Sweden \\ e School of Materials Science and Engineering, University of New South Wales (UNSW), Sydney, 2052, NSW, Australia \\ f School of Engineering, Macquarie University, Sydney, 2109, NSW, Australia \\ *Address correspondence to shujuan.huang@mq.edu.au
}

\begin{abstract}
$\mathrm{PbS}$ quantum dot solar cells (QDSCs) have emerged as a promising low-cost solution processable solar energy harvesting device and demonstrated good air stability and potential for large-scale commercial implementation. PbS QDSC achieved a record certified efficiency of $12 \%$ in 2018 by utilizing an $n+-n-p$ device structure. However, the $p$-type layer has generally suffered from low carrier mobility due to the organic ligand 1,2-ethanedithiol (EDT) that is used to modify the quantum dot (QD) surface. The low carrier mobility of EDT naturally limits the device thickness as the carrier diffusion length is limited by the low mobility. Herein, we improve the properties of the p-type layer through a 2-step hybrid organic ligand treatment. By treating the $p$-type layer with two types of ligands, 3mercaptopropionic acid (MPA) and EDT, the PbS QD surface was passivated by a combination of the two ligands, resulting in an overall improvement in open circuit voltage, fill factor and current density, leading to an improvement in cell efficiency from $7.0 \%$ to a champion cell efficiency of $10.4 \%$. This achievement was a result of improved QD passivation and a reduction in interdot distance, improving charge transport through the $p$ type $\mathrm{PbS}$ quantum dot film.
\end{abstract}

Keywords: PbS quantum dot, quantum dot solar cell, $\mathrm{p}$-type layer, ligand exchange, interdot distance 


\section{Graphical abstract}

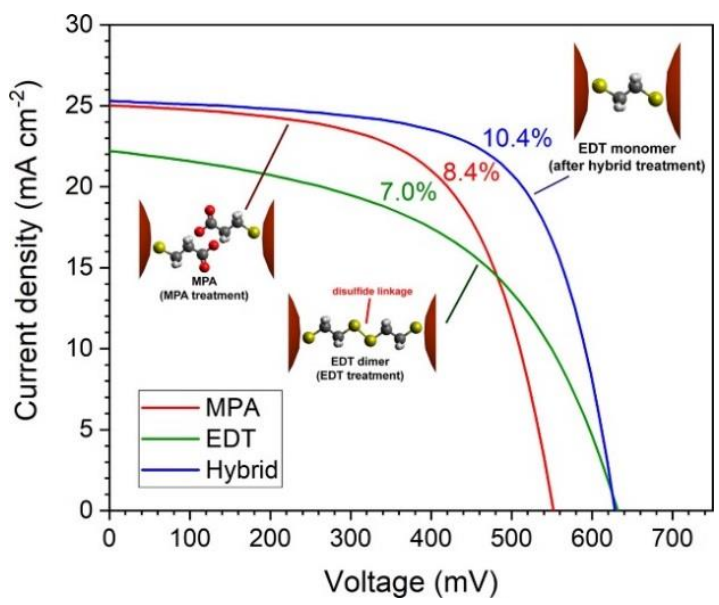

\section{Introduction}

The earliest colloidal quantum dot (CQD) implementation in solar cells achieved an efficiency of $1-3 \%$ based on Schottky junctions ${ }^{1,2}$. Since then, the structure of QDSCs has evolved from a simple metal-PbS-metal Schottky junction, to the current $n^{+}-n-p$ structure ${ }^{3}$. This, along with improvements in the passivation and fabrication of the absorber layer via solution ligand exchange with halide ligands, has allowed the performance of QDSCs to improve to a PCE of $12 \%{ }^{4,5}$. However, challenges remain with the p-type PbS hole transport layer ( $\mathrm{HTL})$, which is a vital component of QDSC devices.

The p-type quantum dot layer based on 1,2-ethanedithiol (EDT) treatment was first demonstrated by Luther et al. in $2008^{6}$. This discovery enabled the formation of $p$-type solids after oxidation in air and formed a film with high electronic coupling that drastically improved electronic properties over PbS QDs capped with oleic acid. This makes EDT treated PbS QD film (PbS-EDT) a suitable HTL that can be implemented into high performance $n^{+}-n-p$ solar cell structures. Various optimizations have been performed on the EDT treatment with adjustments to the ligand concentration and treatment duration and the recipe differs for different publications $5,7,8$. The current highest efficiency cells published have a power conversion efficiency (PCE) of $12.48 \%$ and use PbS-EDT as the $\mathrm{HTL}^{5}$. However, these recent advancements have all been due to progress in improving the passivation of the absorber layer.

To date, there is a lack of progress in improving the p-type PbS-EDT HTL for PbS QDSC. The passivation of PbS QDs with EDT has not fundamentally changed since it was first demonstrated in $2008^{6,9}$. The key to a good HTL is to have sufficiently high p-doping concentration in order to increase the charge carrier concentration and form a wide depletion region when interfaced with an $\mathrm{n}$-type absorber layer. Wider depletion regions can enable thicker devices to increase light harvesting that will boost the current output of the solar cell. One main drawback of PbS-EDT is the low carrier mobility. PbS-EDT thin films have a carrier mobility of around $10^{-4} \mathrm{~cm}^{2} \mathrm{~V}^{-1} \mathrm{~s}^{-1}$, which is one order of magnitude lower than $\mathrm{PbS}$ treated with 3-mercaptopropionic acid (MPA) and two orders of magnitude lower than halide treated PbS QD films ${ }^{10}$. As such, typical methods to increase doping concentration such as the introduction of impurity atoms have limited improvement as the mobility will be drastically reduced due to the impurity atoms. Hu et al. has demonstrated 
that a 1.0\% Ag-doping in PbS-EDT can improve the performance of QDSCs from a PCE of $9.1 \%$ to $10.6 \%$ by improving the hole carrier density and $p$-type character ${ }^{11}$.

While higher Ag doping concentrations further increases hole carrier density, the device performance peaked at $1.0 \%$ doping concentration due to a decrease in carrier mobility and diffusion length that negates the gain from improved charge carrier density. Furthermore, the improved hole mobility is still lower than that of the n-type QD layer. This is an inherent issue with EDT treatment as it has a low carrier mobility due the larger interdot distance. There is an urgent need to find an alternative $\mathrm{p}$-type $\mathrm{PbS}$ material with improved charge transport such that the doping limit can be raised to further improve the charge carrier concentration and reduce carrier recombination velocity at the junction.

An alternative ligand to EDT that contributes a higher carrier mobility is MPA. MPA differs from EDT in that one of the thiol group has been replaced by a carboxylic acid. QD passivated with carboxylic acid ligands have better charge transport compared to thiols ${ }^{12,13}$. Devices using MPA treated PbS quantum dot film (PbS-MPA) as HTL have shown mixed results. PbS-MPA heterojunction devices have demonstrated to have a higher $\mathrm{J}_{s c}$ over PbSEDT devices ${ }^{14}$. However, the gain in $\mathrm{V}_{\mathrm{oc}}$ of PbS-MPA over PbS-EDT did not seem to be reproducible in another study comparing MPA and EDT treatments across various device structures ${ }^{15}$. The lower $\mathrm{V}_{\mathrm{OC}}$ of PbS-MPA over PbS-EDT devices were also observed in this work, although PbS-MPA still demonstrated improvements in Jsc over PbS-EDT. Likewise, majority of PbS QDSC publications prefer EDT over MPA as a p-type ligand due to the superior $\mathrm{V}_{\mathrm{OC}}$ of $\mathrm{PbS}$-EDT based devices, while the $\mathrm{J}_{\mathrm{SC}}$ shortfall is tackled through passivation and thickness improvements in the PbS-Pbl 2 absorber layer ${ }^{4,5,16-19}$, including the world record $12.01 \%$ PCE device ${ }^{5}$.

In this paper, we introduce a novel 2-step MPA and EDT hybrid ligand treatment method to capitalize on the high Jsc characteristic in PbS-MPA devices and high $\mathrm{V}_{\mathrm{OC}}$ characteristic of PbS-EDT devices. The ligand exchange procedure is shown in Figure 1 . The presence of the carboxylic group in MPA from the first treatment would help improve device performance through improvements in the conductivity and mobility by one order magnitude when compared to the thiols ${ }^{10,13,14}$. The two-step treatment also introduces EDT as the primary passivating ligand to achieve a higher $V_{o c}$, while still retaining MPA ligands for improved conductivity over pure PbS-EDT films. This results in an improvement in PCE from 7.0\% for PbS-EDT devices and 8.4\% for PbS-MPA devices to $10.4 \%$ for PbS-Hybrid devices.

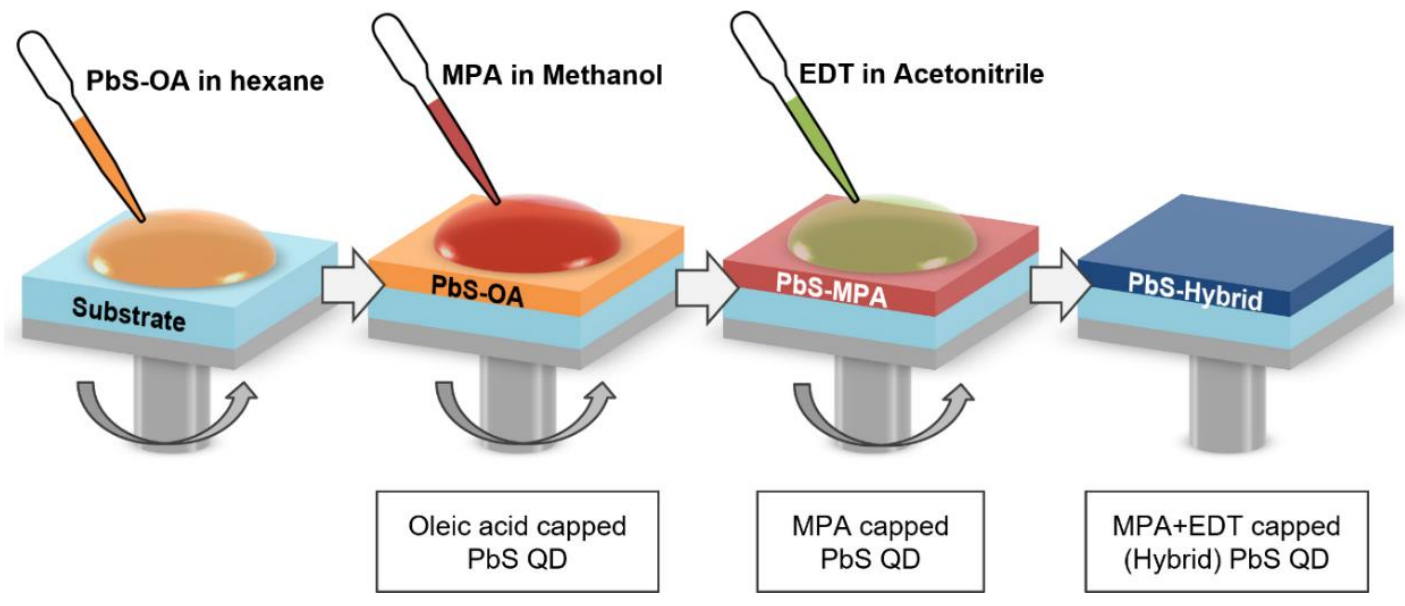


Figure 1: Procedure for 2-step MPA and EDT hybrid ligand treatment. The conventional ligand exchange method for the $p$-type layer involves only one ligand treatment step conducted on an oleic acid capped PbS QD film. The method described in this work demonstrates that a solid layer can also be further treated with a $2^{\text {nd }}$ step to alter and improve the film's properties.

\section{Experimental section}

PbS CQD synthesis: The materials used were lead (II) oxide (PbO) (Sigma-Aldrich 99.999\% trace metals basis), oleic acid (OA) (Sigma-Aldrich 90\% technical grade), 1-octadecene (ODE) (Sigma-Aldrich 90\% technical grade), bis(trimethylsilyl)sulfide (TMS) (Sigma-Aldrich synthesis grade), hexane (Chem-Supply $\geqslant 95 \%$ ) and acetone (Ajax Finechem $\geqslant 99.5 \%$ ). The synthesis of PbS QDs was based on a previous published recipe ${ }^{20}$. The reactants $0.45 \mathrm{~g} \mathrm{PbO}, 1.62 \mathrm{ml}$ $\mathrm{OA}$ and $20 \mathrm{ml}$ ODE were added to a $100 \mathrm{ml}$ three-neck flask and degassed and stirred at $60{ }^{\circ} \mathrm{C}$ for 2 hours and then $100^{\circ} \mathrm{C}$ for 2 hours to form colorless Pb-oleate precursor solution. Once the solution is completely transparent and colorless, $140 \mu \mathrm{lTMS}$ dissolved in $5 \mathrm{ml} \mathrm{ODE}$ was injected into the $\mathrm{Pb}$-oleate solution at $80^{\circ} \mathrm{C}$ and left heating for $10 \mathrm{~s}$ for QD nucleation and growth. After $10 \mathrm{~s}$, the heating mantle was removed and turned off and the solution was allowed to naturally cool to room temperature. The PbS CQD solution was washed and precipitated with hexane and acetone with a volume ratio of 1:3. The precipitation was repeated twice and the PbS CQD was dried under vacuum for 30 minutes.

MgZnO synthesis: The materials used were Zinc acetate dihydrate (Ajax Finechem $\geqslant$ 99.5\%), magnesium acetate tetrahydrate (Sigma-Aldrich $\geqslant 99 \%$ ReagentPlus $^{\circledR}$ ), anhydrous 2-methoxyethanol (Sigma-Aldrich $\geqslant 99.8 \%$ ) and monoethanolamine (Sigma-Aldrich ACS reagent $\geqslant 99.0 \%$ ). $1 \mathrm{~g}$ of zinc acetate dihydrate and $0.05 \mathrm{~g}$ of magnesium acetate tetrahydrate were added to $10 \mathrm{ml}$ of 2-methoxyethanol solvent and $280 \mu \mathrm{l}$ of monoethanolamine complexant. The solution was stirred at $75{ }^{\circ} \mathrm{C}$ for 12 hours until all the precursors were dissolved into a clear homogenous sol-gel.

Device fabrication: The materials used were lead (II) iodide ( $\left.\mathrm{PbI}_{2}\right)$ (Sigma-Aldrich 99\%), dimethylformamide (Analar NORMAPUR 99.9\%), 3-mercaptopropionic acid (Sigma-Aldrich 99\%), methanol ( $\mathrm{RCl}$ Labscan Ltd $\geqslant 99.9 \%), 1$,2-ethanedithiol ( $\mathrm{TCl}>99.0 \%)$, and acetonitrile ( $\mathrm{RCl}$ Labscan Ltd $\geqslant 99.8 \%$ ). The QDSC device fabrication was based on a previous published recipe $^{11}$. ITO glass was cleaned with detergent, deionized water and acetone with ultrasonication. The MgZnO sol-gel was spin-coated on ITO glass substrates at 3000 RPM for $30 \mathrm{~s}$ then dried at $100{ }^{\circ} \mathrm{C}$ for 10 minutes and annealed at $360^{\circ} \mathrm{C}$ for 20 minutes. The $\mathrm{MgZnO}$ film fabrication was repeated 3 times until $\sim 130 \mathrm{~nm}$ thickness was achieved.

For absorber layer, a colloid containing $80 \mu \mathrm{l}$ of PbS CQDs suspended in hexane at $30 \mathrm{mg} / \mathrm{ml}$ concentration was spin-coated on the MgZnO film at 3000 RPM for 30s, treated then with $10 \mathrm{mM} \mathrm{Pbl} 2$ in DMF for 50 s via dip coating, and rinsed with acetonitrile and dried under $\mathrm{N}_{2}$ 
gas flow. This process was repeated 6 times until iodide capped QD (PbS-iodide) absorber layer with 150 $\mathrm{nm}$ thickness was fabricated.

For the HTL layers, $80 \mu \mathrm{l}$ of PbS CQDs with a concentration of $15 \mathrm{mg} / \mathrm{ml}$ suspended in hexane was spin-coated at 3000 RPM for 30s on the substrate. Two ligands were used for this study, MPA and EDT. For PbS-MPA layer, the PbS-OA QD film was treated with $10 \% \mathrm{v} / \mathrm{v}$ MPA in methanol solution for $10 \mathrm{~s}$ followed by a methanol rinse. For PbS-EDT layer, the PbSOA film was treated with $0.02 \% \mathrm{v} / \mathrm{v}$ EDT in acetonitrile solution for $10 \mathrm{~s}$ followed by rinsing with acetonitrile. For the hybrid ligand treated layer (PbS-Hybrid), the same PbS-OA film was treated in 2-steps, i. e. by MPA treatment followed by EDT treatment using the same recipe described above. The fabrication was carried out in air. The PbS QDSC devices were annealed at $75{ }^{\circ} \mathrm{C}$ in air for 15 minutes to oxidize the HTL and help form a p-type film. The devices were stored in a desiccator box in dry air overnight to further facilitate oxidation and improve p-type properties of the HTL in order to achieve the best efficiencies. $100 \mathrm{~nm}$ of gold electrodes were then fabricated on top of the HTL via thermal evaporation. The absorber area of the device was $0.0314 \mathrm{~cm}^{2}$.

Characterization: The optical absorption spectra of the PbS colloid were measured with the Perkin Elmer Lambda1050 UV/Vis/NIR spectrophotometer in ambient condition. The steadystate photoluminescence $(\mathrm{PL})$ spectra of $\mathrm{PbS}$ CQDs dispersed in hexane were measured by QuantaMaster 500 (Horiba) spectrofluorometer with an excitation from a xenon arc lamp at $600 \mathrm{~nm}$ and detected by a InGaAs detector. The Fourier transform infrared spectroscopy (FTIR) measurements were carried out with a PerkinElmer FTIR Spectrometer in reflection mode. The Transmission electron microscopic (TEM) imaging was done on dilute OA-capped PbS QDs dropped onto a copper TEM grid. For treated TEM samples, MPA and EDT ligands with concentrations described in the previous section were dropped onto the copper grid that contains PbS QDs. There samples were then put in an oven for drying to remove any remaining solvents. TEM imaging was carried out on a Philips CM200 field emission TEM. The scanning electron microscopic (SEM) images of the cross section and surface morphology were taken with the FEI Nova NanoSEM 230 Field Emission SEM. The photoemission yield spectroscopy (PYS) was measured using a StelaNet SL3 Deuterium lamp as a light source and JobinYvon HR 250 monochromator with stepper motor to control the wavelength of the incident light, and the amplified photoemission current is measured by grounding the sample stage through a Keithley 617 Electrometer.

The light current density-voltage (J-V) curves of different devices were obtained using a Keithley 2400 (I-V) digital source meter under simulated AM 1.5G solar irradiation at 100 $\mathrm{mW} / \mathrm{cm}^{2}$ (Newport, AAA solar simulator, 94023A-U). Dark J-V measurements were also carried out in the same system using a dark enclosure. The mobility of the HTL was measured with a field effect transistor (FET) using an Agilent B1500A semiconductor characterization system. 


\section{Results and discussion}

3.1 IV performance of solar cell device

(a)

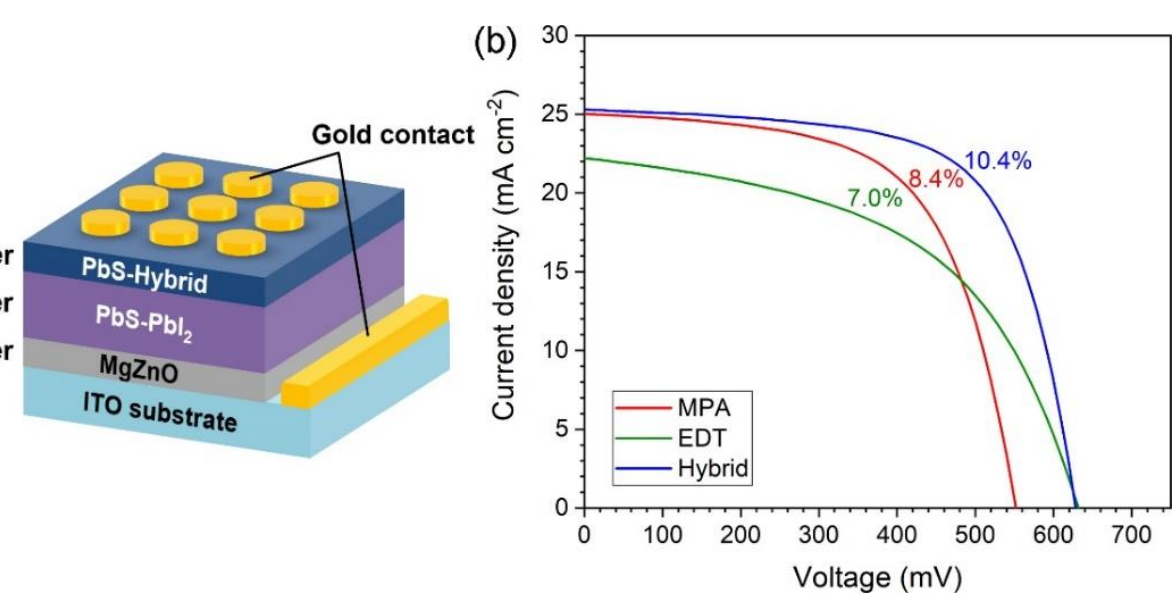

Figure 2: (a) Schematic of the QDSCS. The HTL is the only variable being investigated in this work. (b) The current density-voltage (J-V) curves of champion devices for different HTL ligand treatments. The device with MPA and EDT hybrid ligand treated HTL achieves both high $V_{O C}$ and $J_{S C}$, while the device with EDT treated HTL has a high $V_{O C}$ and MPA treated HTL has a high Jsc.
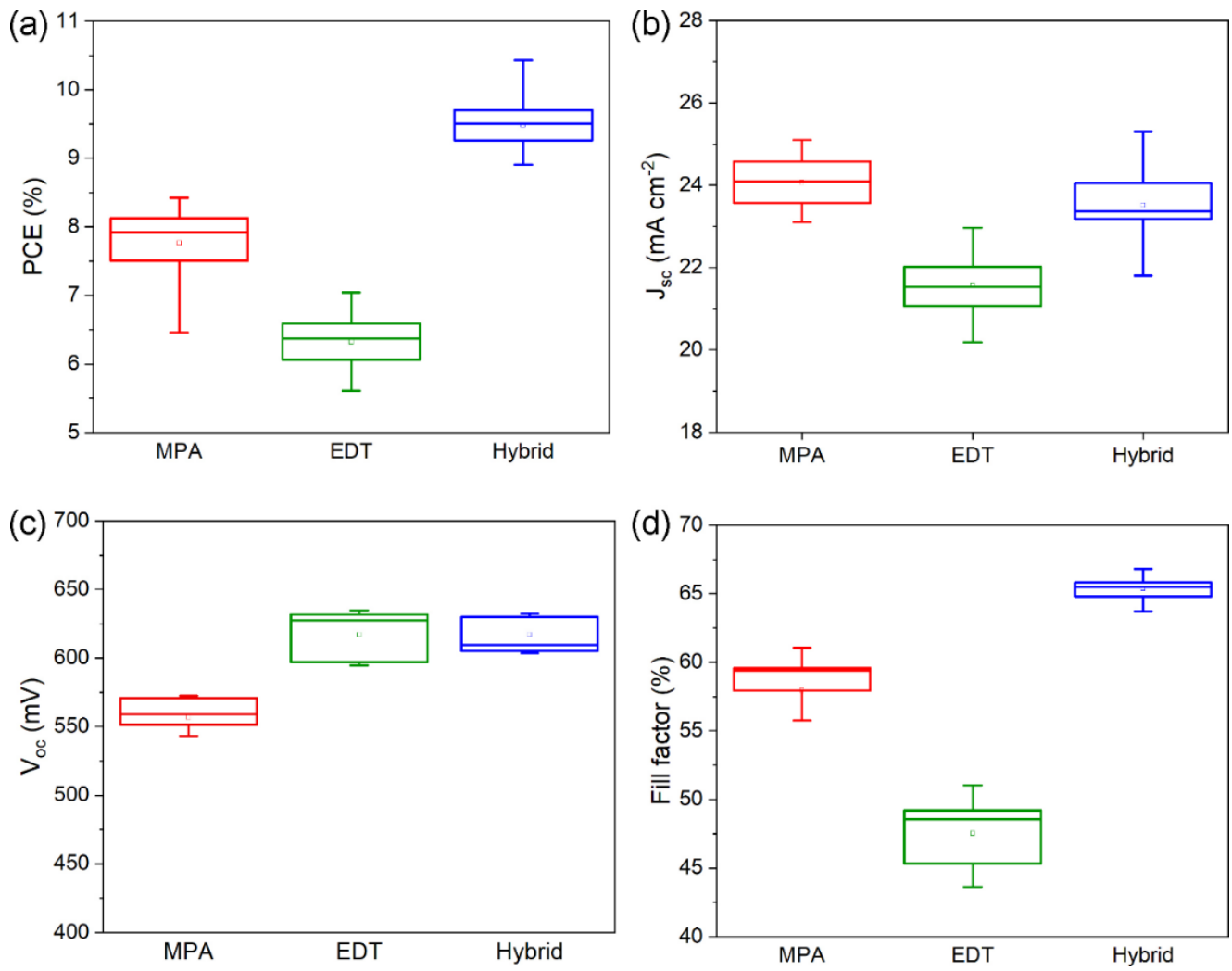
Figure 3: (a) PCE, (b) Jsc, (c) Voc and (d) FF variation between different solar cell devices with different HTLs. The hybrid ligand treatment produces higher performance and reproducibility of the $\mathrm{V}_{\mathrm{Oc}}$ and FF.

\begin{tabular}{|c|c|c|c|c|c|c|}
\hline Treatment & $\begin{array}{c}\mathrm{J}_{\mathrm{SC}} \\
\left(\mathrm{mA} \mathrm{cm}^{-2}\right)\end{array}$ & $\begin{array}{l}V_{o c} \\
(m V)\end{array}$ & $\begin{array}{l}\text { FF } \\
\text { (\%) }\end{array}$ & $\begin{array}{c}R_{S H} \\
\left(\Omega \mathrm{cm}^{2}\right)\end{array}$ & $\begin{array}{c}R_{s} \\
\left(\mathrm{~m} \Omega \mathrm{cm}^{2}\right)\end{array}$ & $\begin{array}{l}\text { PCE } \\
\text { (\%) }\end{array}$ \\
\hline MPA & $\begin{array}{c}24.1 \pm 0.2 \\
(25.1)\end{array}$ & $\begin{array}{c}557 \pm 5 \\
(573)\end{array}$ & $\begin{array}{c}57.9 \pm 1 \\
(61.1)\end{array}$ & $\begin{array}{c}3814 \pm 529 \\
(7004)\end{array}$ & $\begin{array}{c}32 \pm 2 \\
(23)\end{array}$ & $\begin{array}{c}7.8 \pm 0.2 \\
(8.4)\end{array}$ \\
\hline EDT & $\begin{array}{c}21.6 \pm 0.2 \\
(21.9)\end{array}$ & $\begin{array}{c}617 \pm 4 \\
(635)\end{array}$ & $\begin{array}{c}47.5 \pm 0.6 \\
(51.0)\end{array}$ & $\begin{array}{c}2654 \pm 157 \\
(3698)\end{array}$ & $\begin{array}{c}51 \pm 2 \\
(35)\end{array}$ & $\begin{array}{c}6.3 \pm 0.1 \\
(7.0)\end{array}$ \\
\hline Hybrid & $\begin{array}{c}23.5 \pm 0.2 \\
(25.3)\end{array}$ & $\begin{array}{c}617 \pm 3 \\
(633)\end{array}$ & $\begin{array}{c}65.4 \pm 0.2 \\
(66.8)\end{array}$ & $\begin{array}{c}11771 \pm 439 \\
(14064)\end{array}$ & $\begin{array}{c}34 \pm 2 \\
(22)\end{array}$ & $\begin{array}{c}9.5 \pm 0.1 \\
(10.4)\end{array}$ \\
\hline
\end{tabular}

Table 1: Average $\mathrm{J}_{\mathrm{SC}}, \mathrm{V}_{\mathrm{OC}}, \mathrm{FF}, \mathrm{R}_{\mathrm{SH}}, \mathrm{R}_{\mathrm{S}}$ and PCE of solar cell device with different $\mathrm{HTL}$ as described. The values in brackets are the results from the best performing devices.

For device J-V characteristic comparison, two reference cells with PbS-MPA and PbSEDT thin films as HTLs were used as a benchmark against the PbS-Hybrid devices. All other layers of the device are identical. The cross-sectional SEM image of the solar cell device is show in Error! Reference source not found.

From the J-V curves shown in Figure $2 \mathrm{~b}$, it is clear that the PbS-EDT device has a superior $V_{o c}$ at $635 \mathrm{mV}$ compared to PbS-MPA, which is also reflected in other comparison studies between MPA and EDT ${ }^{15,21}$. This is likely due to the higher proportion of thiol groups in EDT that can be easily oxidized to achieve a higher doping concentration during air annealing to form a p-type film. The carboxylic group is inherently more resistant to oxidation and MPA devices have been shown to be more resistant to performance degradation compared to EDT devices when exposed to air [14]. Apart from doping via oxidation, direct addition of thiol ligands to carboxylic acid ligands was also demonstrated to significantly improve the $\mathrm{V}_{\mathrm{OC}}$ of a QD heterojunction solar cell by passivating exposed sulfur atoms on the QD surface ${ }^{22}$. PbS-EDT has also shown to have a longer lifetime than PbS$\mathrm{MPA}^{14}$, possibly reducing carrier recombination.

Note that EDT passivation is effective at a much lower concentration of $0.02 \% \mathrm{v} / \mathrm{v}$, compared to MPA which requires $2 \% \mathrm{v} / \mathrm{v}$ for complete passivation. Based on hard and soft acid base theory (HSAB), thiolate is a soft base and should have a much stronger affinity with the borderline soft acid $\mathrm{Pb}^{2+}$ compared to carboxylate, which is a hard acid ${ }^{23}$. This makes thiols a more potent passivating ligand compared to carboxylic acid.

The shunt resistance for the PbS-EDT device is noticeable lower than the other two devices. From the SEM images as shown in Figure S6, some pin holes can be observed for PbS-EDT but not for PbS-MPA or PbS-Hybrid, which might be the cause of the small $\mathrm{R}_{\text {sh }}$ for PbS-EDT device.

On the other hand, the PbS-MPA device has a better $\mathrm{J}_{\mathrm{Sc}}$ at $25.1 \mathrm{~mA} \mathrm{~cm} \mathrm{~cm}^{-2}$ and a lower Rs of $23 \mathrm{~m} \Omega \mathrm{cm}^{2}$ compared to PbS-EDT. This improvement is consistent with other studies on both heterojunction and $n^{+}-n-p$ device ${ }^{14,15,21}$. The higher $J_{s c}$ is due to the better charge transport of PbS-MPA over PbS-EDT ${ }^{14}$, and higher conductivity has been demonstrated to improve $J_{S C}$ and $R_{S}{ }^{11}$. 
The PbS-Hybrid device successfully combine the characteristics of higher $\mathrm{J}_{s c}$ and lower $\mathrm{Rs}_{\mathrm{S}}$ of PbS-MPA and higher $\mathrm{V}_{\mathrm{OC}}$ of PbS-EDT as shown in Figure 3 and Table 1 . The champion device achieves a PCE of $10.4 \%$ with a $V_{\text {oc }}$ of $633 \mathrm{mV}$, Jsc of $25.3 \mathrm{~mA} \mathrm{~cm}^{-2}$ and FF of $66.8 \%$. The EDT treatment after MPA improves the $V_{O C}$ to EDT levels, indicating improved passivation of the HTL.

Despite the EDT treatment after the MPA treatment, the $\mathrm{J}_{\mathrm{sc}}$ and Rs of PbS-Hybrid are relatively unchanged compared to PbS-MPA, which indicates that PbS-Hybrid device has a similar conductivity, charge generation and collection as PbS-MPA device. External quantum efficiency measurements as shown in Figure $\mathrm{S} 8$ indicates an overall improvement in charge collection for PbS-MPA and PbS-Hybrid devices over PbS-EDT. The high conductivity of PbSHybrid can be attributed to the presence of a small amount of MPA ligands remaining on the PbS QD surface and the shorter interdot distance shown discussed in section 3.3.

\subsection{FTIR spectroscopic study on PbS QD surface passivation}

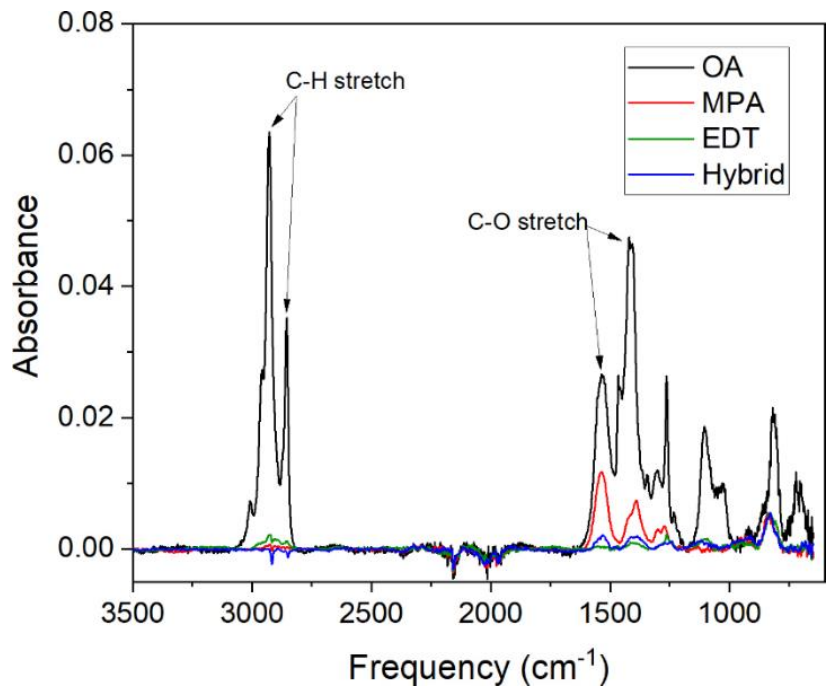

Figure 4: FTIR spectra of PbS QD thin films treated with various ligands. The spectra show the replacement of long chain ligands by short chain linking ligands by tracking the reduction in $\mathrm{C}-\mathrm{H}$ and $\mathrm{C}-\mathrm{O}$ stretch peaks which corresponds to the long carbon chain and carboxylic acid group respectively on OA.

\begin{tabular}{|c|c|c|c|}
\hline \multirow{2}{*}{ Ligand } & \multicolumn{3}{|c|}{ C-O asymmetric stretch } \\
\cline { 2 - 4 } & $\begin{array}{c}\text { Peak } \\
\text { position }\end{array}$ & $\begin{array}{c}\text { Peak } \\
\text { height }\end{array}$ & MPA \% \\
\hline MPA & 1533 & 0.0116 & $100 \%$ \\
\hline Hybrid & 1532 & 0.0020 & $18 \%$ \\
\hline EDT & 1533 & 0.0004 & $0 \%$ \\
\hline
\end{tabular}

Table 2: Percentage of MPA ligand coverage based on FTIR peak position and height of C-O asymmetric peak 
To understand the nature of the hybrid ligand treatment, PbS-MPA, PbS-EDT and PbS-Hybrid thin films were characterized with FTIR spectroscopy. FTIR spectroscopy reveals the presence of organic ligands passivating the PbS QD surface for a dry PbS QD thin film absent of solvents and precursors. As seen from the FTIR spectra in Figure 4, OA molecules are efficiently removed by all ligand treatments. This is evidenced by the significant reduction of $\mathrm{C}-\mathrm{H}$ stretch peaks at $2928 \mathrm{~cm}^{-1}$ and $2856 \mathrm{~cm}^{-1}$ whereby the long carbon chain $\mathrm{OA}$ ligands have been replaced by short carbon chain ligands. There is also a clear reduction of the C-O asymmetric and symmetric stretch peaks at $1533 \mathrm{~cm}^{-1}$ and $1414 \mathrm{~cm}^{-1}$ after treatment with MPA, EDT and hybrid ligand treatment. The MPA and hybrid ligand treatment both retain some $\mathrm{C}-\mathrm{O}$ peak signal due to the presence of carboxylates from the MPA ligand. For PbS-MPA, there are no peaks at 1700 and $1300 \mathrm{~cm}^{-1}$ position, each belonging to the $\mathrm{C}=\mathrm{O}$ stretch and $\mathrm{C}-\mathrm{O}$ stretch peak, indicating that the carboxylic acid is fully deprotonated and the ligand bonds to the QD surface in carboxylate form. For EDT, there is no S-H peak at around $2550 \mathrm{~cm}^{-1}$ which indicates that the ligand is bound in its thiolate form to the QD surface ${ }^{24}$.

Comparing the FTIR spectra of PbS-MPA and PbS-Hybrid, it is clear that hybrid ligand treatment results in the removal of MPA ligands, as evidenced by the significant reduction in both asymmetric and symmetric C-O stretch peaks; the addition of a $2^{\text {nd }}$ step of EDT treatment in the hybrid ligand treatment removes majority of MPA ligands from the QD surface. The ability of EDT to remove and replace MPA ligands is consistent with the HSAB theory discussed previously. This demonstrates that the EDT treatment is effective even for a solid QD thin film linked with short chain MPA ligands.

According to the Beer-Lambert law, absorbance is directly proportional to the concentration of the absorbing species. The percentage of MPA ligands that were replaced by EDT can be estimated by taking the ratio of the $\mathrm{C}-\mathrm{O}$ stretch peak before and after EDT treatment. The $\mathrm{C}-\mathrm{O}$ asymmetric stretch peak at $1531 \mathrm{~cm}^{-1}$ was chosen for the calculation as there is no overlap with EDT peaks in that region. The C-O symmetric stretch peak cannot be used as there is a wide peak at $1434 \mathrm{~cm}^{-1}$ for EDT which is very close to the $1388 \mathrm{~cm}^{-1} \mathrm{C}-\mathrm{O}$ symmetric peak for MPA. As seen in

Table 2, based on the $\mathrm{C}-\mathrm{O}$ asymmetric stretch peak height of PbS-Hybrid, it is estimated that only $18 \%$ of the MPA ligands from the $1^{\text {st }}$ treatment step remains on the surface of the PbS QDs after the $2^{\text {nd }}$ step of EDT treatment, indicating $82 \%$ of the MPA ligands have been replaced by EDT.

The replacement MPA ligands with EDT justifies the improved $\mathrm{V}_{\mathrm{OC}}$ of the PbS-Hybrid device. The built-in voltage of a PbS-EDT device can be improved through surface oxidation by air annealing dithiol treated $\mathrm{PbS}^{9}$. The replacement of MPA ligands with EDT increases the abundance of air sensitive thiol groups for oxidation and this increased oxidation improves the hole doping concentration responsible for the higher $V_{O c}$ of the device.

Considering that most of the ligands in the PbS-Hybrid film are EDT, the reduced presence of MPA ligands may not be sufficient to justify the high $\mathrm{J}_{S C}$ and $\mathrm{R}_{\mathrm{S}}$ characteristics of the PbS-Hybrid device, which match PbS-MPA device performances. This suggests that there are other contributors that can improve the interdot coupling that are independent of the presence of carboxylates such as interdot distance. In summary, the FTIR spectra show a clear replacement of MPA ligands by EDT in the hybrid ligand treatment, demonstrating that 
EDT is the main ligand passivating the PbS QD surface, while about $18 \%$ of MPA ligands remain on the QD surface.

\subsection{TEM study on interdot distance}

Interdot distance has been shown to play a key role in determining the mobility and conductivity of QD thin films. The impact of ligand type and interdot distance to photovoltaic performance has been discussed comprehensively in Wang and co-workers' review paper ${ }^{25}$. Study of different lengths of dithiol ligands on PbSe QDs have shown an exponential increase in mobility as ligand length is reduced ${ }^{26}$. Investigation on $\mathrm{Cu}_{2-x} \mathrm{Se}$ QDs functionalized by dithiol ligands has also shown that the conductivity of $\mathrm{Cu}_{2-x} \mathrm{Se}$ thin films increases as the carbon backbone length is reduced ${ }^{13}$. Under the dithiol family of ligands, EDT is the shortest with only 2 carbon backbone and is unsurprisingly the most commonly used ligand for the HTL in order to maximize film conductivity and mobility.

However, the dithiol ligand has an inherent problem of forming longer chain dimers when exposed to air which reduces mobility and conductivity. Weidman et. al. has observed an unusual trend whereby EDT treated QD film has a larger interparticle spacing of $1.2 \mathrm{~nm}$ than that of longer chain 1,4-butane dithiol treated film with an interparticle spacing of 0.8 $\mathrm{nm}^{27}$. This goes against the otherwise linear decreasing trend of interdot distance with decreasing carbon chain length of the ligand ${ }^{27,28}$. This anomaly in the trend was similarly observed by Lynch et. al. who also observed a detrimental effect on conductivity due to the dimerization of EDT ${ }^{13}$, which is illustrated in

Figure 5a. They proposed that performing the ligand exchange in air will result in the oxidation of the thiol functional group and the formation of sulfur-sulfur bond between two thiols on different QDs, effectively doubling the ligand length, i.e. dimerization. Performing the ligand exchange in an inert environment does not completely eliminate this effect, showing the high sensitivity of EDT even under low oxygen concentration environment ${ }^{13}$. 

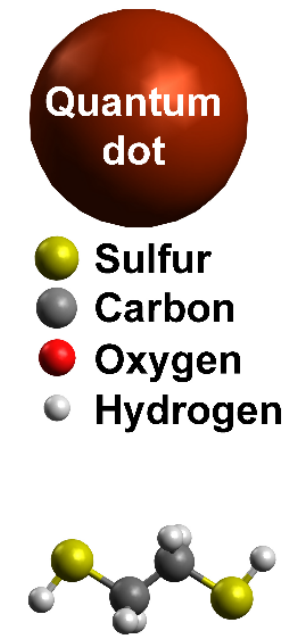

1,2-Ethanedithiol (EDT)

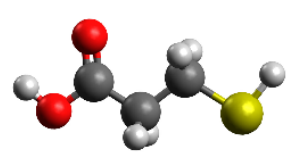

3-mercaptopropionic acid (MPA) (a)

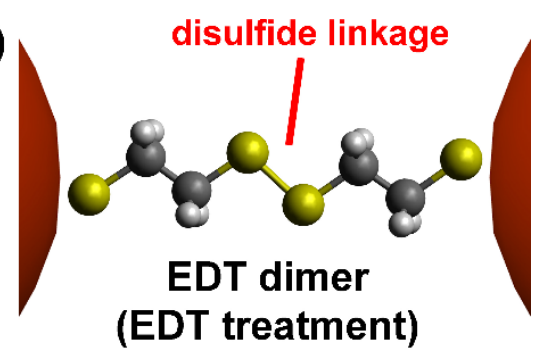

(b)

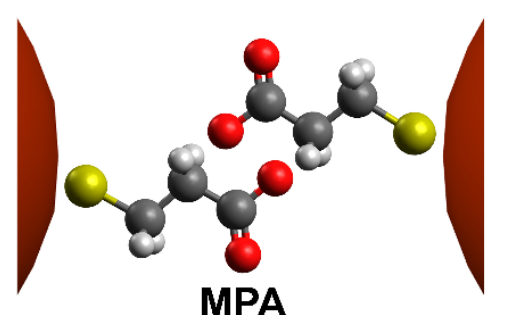

(MPA treatment)

(c)

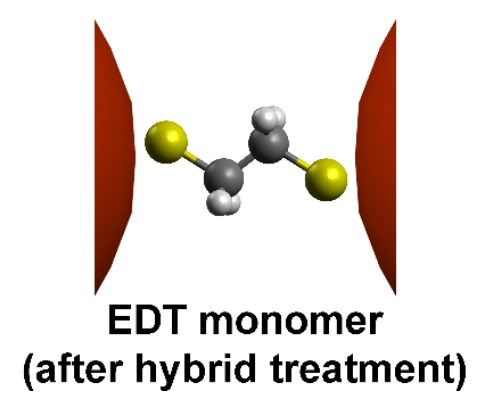

Figure 5: Ligand bonding between QDs for different treatment types and their molecular structures. (a) EDT treatment forms an EDT dimer with a disulfide linkage. (b) MPA treatment forms two overlapping MPA ligands. (c) Hybrid ligand treatment forming the proposed EDT monomer bond. The short interdot distance for the hybrid ligand is likely due to bridging by only one EDT monomer as opposed to the EDT dimer for EDT treated PbS QDs.

To understand the impact of hybrid ligand treatment to the interdot distance using TEM imaging, slightly larger QDs i.e. $4.5 \mathrm{~nm}$ in diameter were synthesized and treated with the appropriate ligands and observed under the TEM. The QDs used were larger than those synthesized for QDSC device in order to facilitate clear imaging. The larger QDs were synthesized by increasing the OA:PbO mole ratio from 2.5:1 to $14: 1$, which yields a QD size of $4.5 \mathrm{~nm}$ with a narrow size distribution as shown in Error! Reference source not found. After the images were taken as shown in

Figure 6, the QD diameter and QD centre-to-centre distance were measured using an algorithm written in python and the OpenCV software packages. The interdot distance is the difference between the average centre-to-centre distance and the average diameter as shown in Error! Reference source not found. 

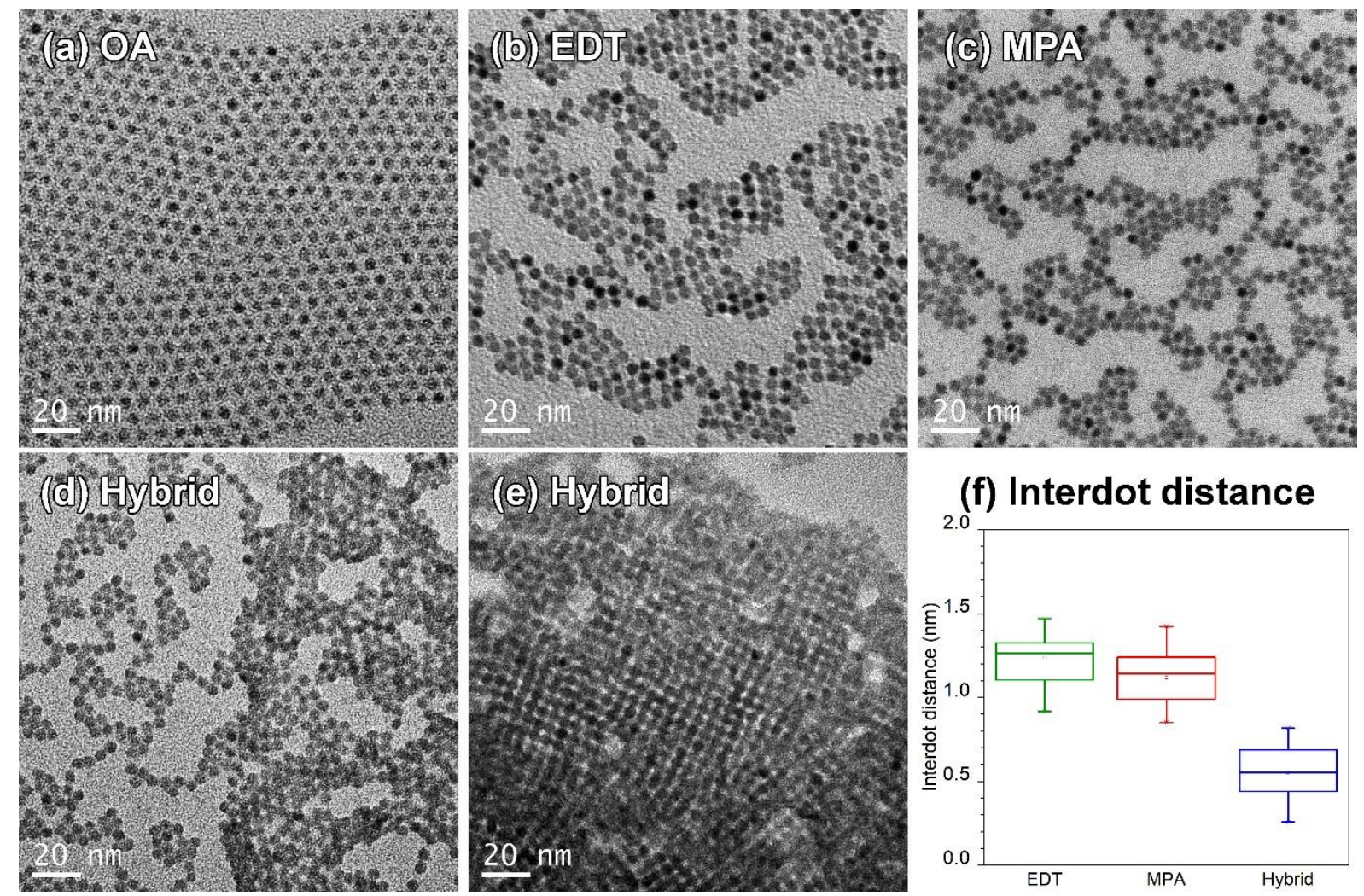

Figure 6: TEM images of $4.5 \pm 0.6 \mathrm{~nm}$ PbS CQDs functionalized by the ligands of (a) OA, (b) $E D T$, (c) MPA, and (d) and (e) hybrid ligands. The measured interdot distance is $2.9 \pm 0.4 \mathrm{~nm}$ for PbS-OA, $1.2 \pm 0.1 \mathrm{~nm}$ for PbS-EDT, $1.1 \pm 0.2 \mathrm{~nm}$ for PbS-MPA, and $0.6 \pm 0.2 \mathrm{~nm}$ for PbSHybrid. (e) shows PbS QDs multilayered superlattice matrix after hybrid ligand treatment with highly ordered self-assembled regions, which dominates most of the TEM imaging of the hybrid ligand samples. Such dense regions were not found in the PbS-EDT and PbS-MPA samples. (f) shows the interdot distance between PbS QDs treated with EDT, MPA and hybrid ligands. The PbS-Hybrid QDs show the shortest interdot distance as the QDs are only bridge by one EDT monomer as oppossed to an EDT dimer for PbS-EDT sample.

\section{From}

Figure 6fError! Reference source not found., PbS-EDT QDs show the largest interdot distance of $1.2 \pm 0.1 \mathrm{~nm}$ after exchanging the original ligands of OA by EDT, which matches literature ${ }^{27}$. This value is double the molecular length of EDT, suggesting EDT molecules are dimerized as shown in Figure 5a. The interdot distance of PbS-MPA sample is $1.1 \pm 0.2 \mathrm{~nm}$, consistent with literature ${ }^{27}$. The molecule length of MPA was calculated to be $6.7 \AA$ from software simulations using the MMFF94Fs model for energy minimization ${ }^{29}$. This implies that the QDs in PbS-MPA film are separated by two MPA molecules as shown in Figure 5b.

As can be seen in

Figure $6 f$, the hybrid treatment significantly reduces the interdot distance to $0.6 \pm$ $0.2 \mathrm{~nm}$. Since the hybrid treatment is a 2-step treatment, i.e. an MPA treatment followed by an EDT treatment, the interdot distance is firstly reduced from $2.9 \mathrm{~nm}$ for PbS-OA to $1.1 \mathrm{~nm}$ with the 1st MPA ligand treatment. At this point, the QDs are separated by two MPA molecular lengths. The 2 nd step of the treatment with EDT introduces a slightly shorter 
ligand with a length of $6.1 \AA$ to further reduce the interdot distance by displacing MPA. Unlike the direct treatment of EDT on PbS-OA, treating PbS-MPA QDs with EDT does not lead to EDT dimerization that is responsible for the large interdot distance, allowing the QDs to directly couple by just one EDT ligand length as shown in Figure $5 \mathrm{c}$. It is likely that the more densely packed PbS-MPA layer prevents the diffusion of excessive amounts of EDT ligands between QDs that drives the oxidation of thiols to disulfides. The reduction in interdot distance for PbS-Hybrid helps enhance interdot coupling and improves the mobility and conductivity of hybrid ligands over longer interdot distance such as PbS-EDT. This mobility and conductivity gains translate into the $J_{S C}$ and $R_{s}$ improvements of the QDSC devices using PbS-Hybrid as the HTL over PbS-EDT which will be elaborated in a later section.

The hybrid ligand treatment also yields a densely packed and highly self-assembled 3D structures shown in

Figure 6e that are not seen in the TEM images of MPA or EDT treated PbS QD film. This demonstrates that the reduced interdot distance by the hybrid ligand treatment allows for high degree of ordering. Single ligand treatment of PbS QDs tend to yield localize short range ordering of QDs separated by empty space, resembling a web of QDs such as the ones shown in

Figure $6 \mathrm{~b}$ and $\mathrm{c}$, which drastically reduces charge transport throughout the $\mathrm{PbS} Q \mathrm{QD}$ film.

\subsection{Conductivity and mobility}

To observe the changes in the conductivity of PbS QD film with different treatment, a Schottky junction with an ITO/PbS/Au device structure was fabricated and the dark J-V was measured. The resistance was determined from the slope of the J-V curve at current $=0$. The thickness of the device was estimated to be $70 \mathrm{~nm}$ and the device area is $0.0314 \mathrm{~cm}^{2}$. The conductivity was then calculated from the following equation:

$$
\frac{1}{\sigma}=\rho=R \frac{A}{l}
$$

where $\sigma$ is conductivity, $\rho$ is resistivity, $R$ is resistance, $A$ is area of the device, and $l$ is thickness of the device. 


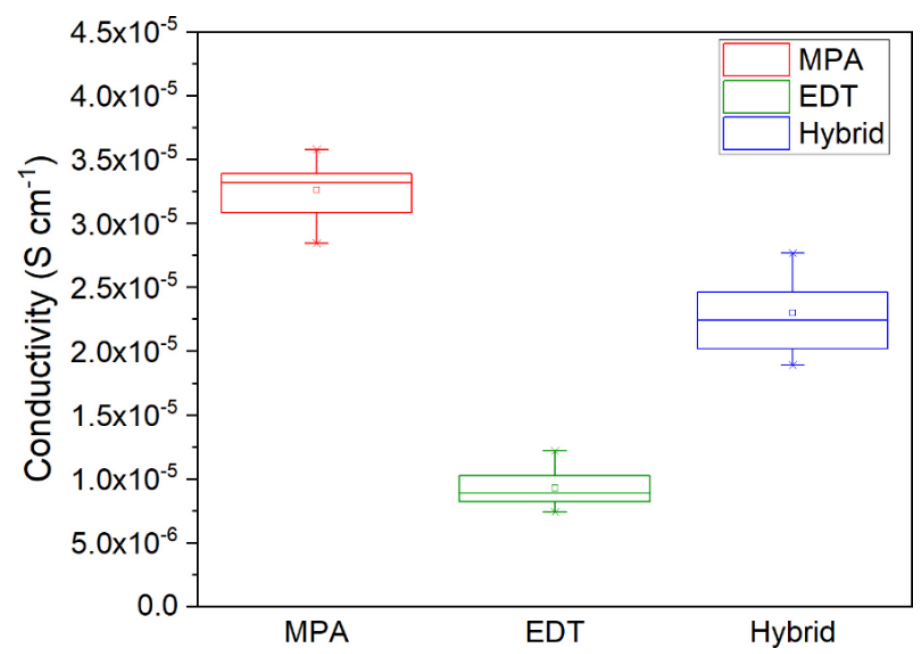

Figure 7: Conductivity of PbS QDs treated with MPA, EDT and Hybrid ligands

The conductivity of the PbS QD film treated by hybrid ligands lies between EDT and MPA-treated samples, as shown in Figure 7. This is a marked improvement over EDT despite the PbS-Hybrid containing $82 \%$ EDT ligands as inferred from the FTIR spectra. This confirms that the reduction in interdot distance resulted in an improvement to the conductivity of the film. However, the increase in conductivity falls short of PbS-MPA, indicating that the functional group on the QD surface plays an equally significant role in determining the conductivity. The conductivity trends well with the Jsc performance of the three devices, further confirming the impact of conductivity towards Jsc.

\begin{tabular}{ccc}
\hline Mreatment & $\begin{array}{c}\text { Mobility } \\
\left(\mathrm{cm}^{\mathbf{2}} \mathbf{~ V}^{-\mathbf{1}} \mathbf{s}^{-1}\right)\end{array}$ & Reference \\
\hline MPA & 0.00510 & 14 \\
EDT & 0.00018 & this work \\
Hybrid & 0.00120 & this work \\
\hline
\end{tabular}

Table 3: Mobility of PbS QDs treated with MPA, EDT and Hybrid ligands

To further identify the contribution towards improved conductivity, the mobility of $\mathrm{PbS}$-Hybrid and PbS-EDT films were measured by fabricating a PbS thin film field effect transistor and the results are shown in Table 3. Similar to the conductivity trend, the mobility of PbS-Hybrid is in between PbS-MPA and PbS-EDT, suggesting that the mobility is likely the main contributor to the improvement in conductivity of the film.

There are possibly two contributions towards the improvement of conductivity and mobility of PbS-Hybrid sample. First is the reduction in the interdot distance compared to $\mathrm{PbS}$-EDT sample due to the lack of dimerization of EDT. The shorter interdot distance can increase carrier tunnelling probability between QDs hence improving film's mobility and conductivity ${ }^{26,28}$. Secondly, the mobility is also enhanced due to the presence of small amounts of the carboxylate functional group from the remaining MPA ligands as shown by the FTIR results. The mobility and conductivity of QDs capped with carboxylic acids ligands with similar ligand lengths have been previously reported to be superior to those capped by thiols ${ }^{12,13}$. 


\subsection{Ionisation energy}

The improved $\mathrm{J}_{\mathrm{Sc}}$ of PbS-Hybrid can be further interpreted from the changes to the valence band edge of the $\mathrm{p}$-type PbS films. The valence band edge of PbS-EDT, PbS-MPA and $\mathrm{PbS}-\mathrm{Hybrid}$ is determined from the ionization energy of the film. The ionization energy was measured using photoelectron yield spectroscopy (PYS), which is suitable for quantum dots $^{30}$ and ultra-thin buffer layers ${ }^{31}$. This method is especially suitable for ligand exchanged p-type quantum dots with low mobility and thickness.

\begin{tabular}{|c|c|}
\hline Sample & $\begin{array}{c}\text { Ionisation energy } \\
(\mathrm{eV})\end{array}$ \\
\hline PbS-EDT & 4.94 \\
\hline PbS-MPA & 4.87 \\
\hline PbS-Hybrid & 4.85 \\
\hline
\end{tabular}

Table 4: Ionisation energy measured using PYS

For the PbS-Hybrid film, the ionisation energy closely matches with that of MPA as shown in Table 4. This contrasts with the large reduction in MPA ligands shown in the FTIR results. The PbS-Hybrid film retains the ionisation energy of MPA with the presence of just a minority amount of MPA. When there are two species of ligands on the surface, likely additional shallower valence states from the MPA ligand contribute to the overall shallower ionisation energy of the PbS-Hybrid sample. The shallower ionisation energy of PbS-MPA and PbS-Hybrid prevents the backflow of holes into the absorber layer, thus improving the hole transport properties and reducing recombination. With a lower recombination, both PbS-MPA and PbS-Hybrid devices show enhanced Jsc over PbS-EDT.

Note that the ionisation energy of all species is about 0.2-0.3 eV shallower compared to the reported value measured by UV photoelectron spectroscopy (UPS) ${ }^{32}$, perhaps due to the inherent charging problem for samples measured with UPS. However, both the measurements here and in literature show a similar trend of MPA having a shallower ionization potential ${ }^{32}$. Comparison between oleic acid and octadecyl thiol also show a similar $0.05 \mathrm{eV}$ reduction in ionization energy ${ }^{30}$.

\section{Conclusion}

This work demonstrates a novel method to produce closely packed p-type QD thin films with a short interdot distance of $\sim 0.6 \mathrm{~nm}$ using the 2-step hybrid ligand treatment. This is the first observation of a $0.6 \mathrm{~nm}$ interdot distance with EDT as a dominant ligand, as confirmed by TEM imaging. EDT ligands have previously been only observed in its dimer form with an interdot distance of $1.2 \mathrm{~nm}$. The 2-step hybrid ligand exchange works by using MPA as an intermediate ligand to prevent dimerization of the EDT ligands during the EDT ligand exchange step. This yields a film that has improved interdot coupling and conductivity. As shown in the J-V results, the devices based on PbS-Hybrid HTL have demonstrated significant improvement in $\mathrm{J}_{\mathrm{Sc}}$ and $\mathrm{FF}$ compared to that of the PbS-EDT device, while the $\mathrm{V}_{\mathrm{O}}$ and FF have improved compared to that of PbS-MPA devices. The best 
performing PbS-Hybrid device has achieved a PCE of 10.4\% which demonstrates an improvement by $23 \%$ over PbS-MPA device (PCE of $8.4 \%$ ) and $48 \%$ compared to PbS-EDT device (PCE of 7\%).

\section{Acknowledgements}

This Program was supported by the Australian Government through the Australian Renewable Energy Agency and Australian Research Council. Responsibility for the views, information or advice expressed herein is not accepted by the Australian Government. Authors would also like to thank the Electron Microscopy Unit of UNSW for the microscopy imaging support.

\section{References}

(1) Luther, J. M.; Law, M.; Beard, M. C.; Song, Q.; Reese, M. O.; Ellingson, R. J.; Nozik, A. J. Schottky Solar Cells Based on Colloidal Nanocrystal Films. Nano Lett. 2008, 8 (10), 3488-3492.

(2) Johnston, K. W.; Pattantyus-Abraham, A. G.; Clifford, J. P.; Myrskog, S. H.; MacNeil, D. D.; Levina, L.; Sargent, E. H. Schottky-Quantum Dot Photovoltaics for Efficient Infrared Power Conversion. Appl. Phys. Lett. 2008, 92 (15), 151115-122111.

(3) Chuang, C.-H. M.; Brown, P. R.; Bulović, V.; Bawendi, M. G. Improved Performance and Stability in Quantum Dot Solar Cells through Band Alignment Engineering. Nat. Mater. 2014, 13 (8), 796-801.

(4) Liu, M.; Voznyy, O.; Sabatini, R.; García De Arquer, F. P.; Munir, R.; Balawi, A. H.; Lan, X.; Fan, F.; Walters, G.; Kirmani, A. R.; et al. Hybrid Organic-Inorganic Inks Flatten the Energy Landscape in Colloidal Quantum Dot Solids. Nat. Mater. 2017, 16 (2), 258263.

(5) Xu, J.; Voznyy, O.; Liu, M.; Kirmani, A. R.; Walters, G.; Munir, R.; Abdelsamie, M.; Proppe, A. H.; Sarkar, A.; García de Arquer, F. P.; et al. 2D Matrix Engineering for Homogeneous Quantum Dot Coupling in Photovoltaic Solids. Nature Nanotechnology. 2018, pp 1-7.

(6) Luther, J. M.; Law, M.; Song, Q.; Perkins, C. L.; Beard, M. C.; Nozik, A. J. Structural, Optical, and Electrical Properties of Self-Assembled Films of PbSe Nanocrystals Treated with 1,2-Ethanedithiol. ACS Nano 2008, 2 (2), 271-280.

(7) Lu, K.; Wang, Y.; Liu, Z.; Han, L.; Shi, G.; Fang, H.; Chen, J.; Ye, X.; Chen, S.; Yang, F.; et al. High-Efficiency PbS Quantum-Dot Solar Cells with Greatly Simplified Fabrication Processing via "Solvent-Curing." Advanced Materials. Wiley-Blackwell May 2, 2018, p 1707572.

(8) Stavrinadis, A.; Pradhan, S.; Papagiorgis, P.; Itskos, G.; Konstantatos, G. Suppressing Deep Traps in PbS Colloidal Quantum Dots via Facile lodid[1] A. Stavrinadis, S. Pradhan, P. Papagiorgis, G. Itskos, and G. Konstantatos, "Suppressing Deep Traps in PbS Colloidal Quantum Dots via Facile lodide Substitutional Doping for Solar Ce. ACS Energy Lett. 2017, 2 (4), 739-744.

(9) Klem, E. J. D.; Shukla, H.; Hinds, S.; MacNeil, D. D.; Levina, L.; Sargent, E. H. Impact of Dithiol Treatment and Air Annealing on the Conductivity, Mobility, and Hole Density in PbS Colloidal Quantum Dot Solids. Appl. Phys. Lett. 2008, 92 (21), 212105.

(10) Tang, J.; Kemp, K. W.; Hoogland, S.; Jeong, K. S.; Liu, H.; Levina, L.; Furukawa, M.; Wang, X.; Debnath, R.; Cha, D.; et al. Colloidal-Quantum-Dot Photovoltaics Using 
Atomic-Ligand Passivation. Nat. Mater. 2011, 10 (10), 765-771.

(11) Hu, L.; Zhang, Z.; Patterson, R. J.; Hu, Y.; Chen, W.; Chen, C.; Li, D.; Hu, C.; Ge, C.; Chen, Z.; et al. Achieving High-Performance PbS Quantum Dot Solar Cells by Improving Hole Extraction through Ag Doping. Nano Energy 2018, 46, 212-219.

(12) Zarghami, M. H.; Liu, Y.; Gibbs, M.; Gebremichael, E.; Webster, C.; Law, M. P-Type $\mathrm{PbSe}$ and PbS Quantum Dot Solids Prepared with Short-Chain Acids and Diacids. ACS Nano 2010, 4 (4), 2475-2485.

(13) Lynch, J.; Kotiuga, M.; Doan-Nguyen, V. V. T.; Queen, W. L.; Forster, J. D.; Schlitz, R. A.; Murray, C. B.; Neaton, J. B.; Chabinyc, M. L.; Urban, J. J. Ligand Coupling Symmetry Correlates with Thermopower Enhancement in Small-Molecule/Nanocrystal Hybrid Materials. ACS Nano 2014, 8 (10), 10528-10536.

(14) Jeong, K. S.; Tang, J.; Liu, H.; Kim, J.; Schaefer, A. W.; Kemp, K.; Levina, L.; Wang, X.; Hoogland, S.; Debnath, R.; et al. Enhanced Mobility-Lifetime Products in PbS Colloidal Quantum Dot Photovoltaics. ACS Nano 2012, 6 (1), 89-99.

(15) Crisp, R. W.; Kroupa, D. M.; Marshall, A. R.; Miller, E. M.; Zhang, J.; Beard, M. C.; Luther, J. M. Metal Halide Solid-State Surface Treatment for High Efficiency PbS and PbSe QD Solar Cells. Sci. Rep. 2015, 5 (1), 9945.

(16) Lan, X.; Voznyy, O.; Kiani, A.; García De Arquer, F. P.; Abbas, A. S.; Kim, G. H.; Liu, M.; Yang, Z.; Walters, G.; Xu, J.; et al. Passivation Using Molecular Halides Increases Quantum Dot Solar Cell Performance. Adv. Mater. 2016, 28 (2), 299-304.

(17) Pradhan, S.; Stavrinadis, A.; Gupta, S.; Bi, Y.; Di Stasio, F.; Konstantatos, G. Trap-State Suppression and Improved Charge Transport in PbS Quantum Dot Solar Cells with Synergistic Mixed-Ligand Treatments. Small 2017, 13 (21), 1700598.

(18) Azmi, R.; Sinaga, S.; Aqoma, H.; Seo, G.; Ahn, T. K.; Park, M.; Ju, S. Y.; Lee, J. W.; Kim, T. W.; Oh, S. H.; et al. Highly Efficient Air-Stable Colloidal Quantum Dot Solar Cells by Improved Surface Trap Passivation. Nano Energy 2017, 39, 86-94.

(19) Cao, Y.; Stavrinadis, A.; Lasanta, T.; So, D.; Konstantatos, G. The Role of Surface Passivation for Efficient and Photostable PbS Quantum Dot Solar Cells. Nat. Energy 2016, 1 (4), 16035.

(20) Hu, L.; Li, D. B.; Gao, L.; Tan, H.; Chen, C.; Li, K.; Li, M.; Han, J. B.; Song, H.; Liu, H.; et al. Graphene Doping Improved Device Performance of $\mathrm{ZnMgO/PbS}$ Colloidal Quantum Dot Photovoltaics. Adv. Funct. Mater. 2016, 26 (12), 1899-1907.

(21) Zhang, Y.; Ding, C.; Wu, G.; Nakazawa, N.; Chang, J.; Ogomi, Y.; Toyoda, T.; Hayase, S.; Katayama, K.; Shen, Q. Air Stable PbSe Colloidal Quantum Dot Heterojunction Solar Cells: Ligand-Dependent Exciton Dissociation, Recombination, Photovoltaic Property, and Stability. 2016.

(22) Kim, S.; Im, S. H.; Kang, M.; Heo, J. H.; Seok, S. II; Kim, S.-W.; Mora-Seró, I.; Bisquert, J. Air-Stable and Efficient Inorganic-Organic Heterojunction Solar Cells Using PbS Colloidal Quantum Dots Co-Capped by 1-Dodecanethiol and Oleic Acid. Phys. Chem. Chem. Phys. 2012, 14 (43), 14999.

(23) Lin, Q.; Yun, H. J.; Liu, W.; Song, H. J.; Makarov, N. S.; Isaienko, O.; Nakotte, T.; Chen, G.; Luo, H.; Klimov, V. I.; et al. Phase-Transfer Ligand Exchange of Lead Chalcogenide Quantum Dots for Direct Deposition of Thick, Highly Conductive Films. J. Am. Chem. Soc. 2017, 139 (19), 6644-6653.

(24) Lefrançois, A.; Couderc, E.; Faure-Vincent, J.; Sadki, S.; Pron, A.; Reiss, P. Effect of the Treatment with (Di-)Amines and Dithiols on the Spectroscopic, Electrochemical and Electrical Properties of CdSe Nanocrystals' Thin Films. J. Mater. Chem. 2011, 21 (31), 
11524.

(25) Wang, R.; Shang, Y.; Kanjanaboos, P.; Zhou, W.; Ning, Z.; Sargent, E. H. Colloidal Quantum Dot Ligand Engineering for High Performance Solar Cells. Energy and Environmental Science. Royal Society of Chemistry April 1, 2016, pp 1130-1143.

(26) Liu, Y.; Gibbs, M.; Puthussery, J.; Gaik, S.; Ihly, R.; Hillhouse, H. W.; Law, M. Dependence of Carrier Mobility on Nanocrystal Size and Ligand Length in Pbse Nanocrystal Solids. Nano Lett. 2010, 10 (5), 1960-1969.

(27) Weidman, M. C.; Yager, K. G.; Tisdale, W. A. Interparticle Spacing and Structural Ordering in Superlattice Pbs Nanocrystal Solids Undergoing Ligand Exchange. Chem. Mater. 2015, 27 (2), 474-482.

(28) Huang, S.; Tsutsui, G.; Sakaue, H.; Shingubara, S.; Takahagi, T. Control of Interdot Space and Dot Size in a Two-Dimensional Gold Nanodot Array. Jpn. J. Appl. Phys. 1999, 38 (Part 2, No. 4B), L473-L476.

(29) Halgren, T. A. MMFF VI. MMFF94s Option for Energy Minimization Studies. J. Comput. Chem. 1999, 20 (7), 720-729.

(30) Jasieniak, J.; Califano, M.; Watkins, S. E. Size-Dependent Valence and Conduction Band-Edge Energies of Semiconductor Nanocrystals. In ACS Nano; 2011; Vol. 5, pp 5888-5902.

(31) Gentle, A. R.; Smith, G. B.; Watkins, S. E. Discharge Amplified Photo-Emission from Ultra-Thin Films Applied to Tuning Work Function of Transparent Electrodes in Organic Opto-Electronic Devices. Appl. Surf. Sci. 2013, 285, 110-114.

(32) Brown, P. R.; Kim, D.; Lunt, R. R.; Zhao, N.; Bawendi, M. G.; Grossman, J. C.; Bulović, V. Energy Level Modification in Lead Sulfide Quantum Dot Thin Films through Ligand Exchange. ACS Nano 2014, 8 (6), 5863-5872. 